Martin T, Sauleau E, Velten M, Sibilia J. Arthritis Rheumatol. 2016;68:1731-7.

Disclosure of Interests: None declared

DOI: 10.1136/annrheumdis-2019-eular.5556

\section{FRI0338 ANTI-MDA5 IDIOPATHIC INFLAMMATORY MYOSITIS (IIM) CONFERS POOR PROGNOSIS BUT NEGATIVE MYOSITIS SPECIFIC ANTIBODY (MSA) IS NOT BENIGN EITHER}

Sin Ngai Ng, Chun Man Ng, Chu Oi Ciang, Moon Ho Leung. Queen Elizabeth Hospital, Medicine, Hong Kong, Hong Kong (SAR)

Background: MSA test is useful to diagnose IIM and subcategorize patients by disease phenotypes.

Objectives: The study aims to evaluate the survival of IIM patients of different MSA patterns.

Methods: An IIM registry had been set up in a tertiary referral centre since 2014 by recruiting prevalent and incident cases. Patients were followed-up prospectively. This study included patients fulfilling the 2017 EULAR/ACR classification criteria for $\mathrm{IIM}^{1}$ and excluded those aged $<18$ at disease onset. Immunoblot EUROLINE autoimmune inflammatory myopathies 16 antigens strip (EUROIMMUNE AG, Lubeck, Germany) was used. Information including baseline demographic data, disease manifestations, MSA results, co-existing malignancy, duration of survival and causes of death were collected. IIM patients were divided into seven groups, which included 1) anti-aminoacyl tRNA synthetase (ARS) 2) antiMDA5, 3) anti-TIF1 $\gamma /$ anti-NXP2, 4) double positive MSA, 5) other MSAs, 6) negative MSA/MAA (myositis associated antibodies) and 7) positive MAA only. Survival probabilities were compared among different MSA groups by using the Kaplan-Meier method and log-rank test. A two-tailed probability value $(p)<0.05$ was considered significant. The study was approved by Kowloon Central Cluster Ethic Committee (ref.: KC/KE-170177/ER-3)

Results: Among 112 IIM patients, 79 (70.5\%) were female, and the median age of onset was 55 (18-90) years old; $63.4 \%$ were dermatomyositis (DM), $17.9 \%$ polymyositis (PM) and $18.8 \%$ clinically amyotrophic DM (CADM). Co-existing interstitial lung disease (ILD) was common and found in $65(58 \%)$ patients; $16(14.3 \%)$ had rapidly progressive interstitial lung disease (RPILD), and $16(14.3 \%)$ died within the observed period. Overall, the commonest cause of death was RPILD, followed by infection and malignancy. While anti-MDA5 was strongly associated with RPILD (odds ratio $=33.0[95 \% \mathrm{Cl}: 7.2-151.8], \mathrm{p}<0.001)$, anti-MDA5 group had the worst survival, with 1 -year and 5 -year survival both at $43 \%$, compared to above $80 \%$ in all other groups (log-rank test $p<0.001$ ) (Table 1). There were nine patients with double positive MSA and 28 had negative MSA/ MAA. Analysis between MSA sub-groups found that the double positive MSA, MAA only and other MSAs group had no mortality during study period while the negative MSA/MAA group had the second highest mortality following the anti-MDA5 group (Figure 1). Infection and malignancy were the two major causes of death in the MSA/MAA negative group. Conclusion: Anti-MDA5 associated RPILD was the leading cause of mortality in IIM. However, those tested negative for both MSA and MAA by current immunoblot technique also had guarded prognosis related to the risk of infection and malignancy.

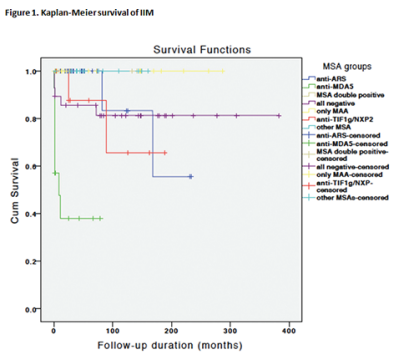

Figure 1. Kaplan-Meier survival of IIM
Table 1. Survival rates in different MSA groups

\begin{tabular}{lcc}
\hline Survival & $\mathbf{1}$ year & $\mathbf{5}$ years \\
\hline Anti-ARS & $100 \%$ & $92 \%$ \\
Anti-MDA5 & $43 \%$ & $43 \%$ \\
Double positive & $100 \%$ & $100 \%$ \\
Negative MSA/MAA & $89 \%$ & $82 \%$ \\
Only positive MAA & $100 \%$ & $100 \%$ \\
Anti-TIF1 $\gamma /$ anti-NXP2 & $100 \%$ & $88 \%$ \\
Other MSAs & $100 \%$ & $100 \%$ \\
\hline
\end{tabular}

\section{REFERENCES:}

[1] Lundberg IE, et al.. ARD 2017;76(12):1955-1964.

Disclosure of Interests: None declared

DOI: 10.1136/annrheumdis-2019-eular.3185

\section{FRI0339 CLINICAL CHARACTERISTICS OF ANTI-RO52A AND ANTI-RO52B ANTIBODIES IN DERMATOMYOSITIS/ POLYMYOSITIS}

Mariko Ogawa-Momohara, Yoshinao Muro, Masashi Akiyama. Nagoya University Graduate School of Medicine, dermatology, Nagoya, Japan

Background: The anti-Ro52 antibody, found in numerous systemic autoimmune conditions, is one of the most common autoantibodies in inflammatory myositis. It is known to be associated with interstitial lung disease (ILD) and to coexist with anti-Jo- $1^{1}$. There are two spliced forms: Ro52 $\alpha$ and Ro52 $\beta$. Ro52 $\beta$ was originally reported in 1995 as a spliced form of Ro52 $\alpha$ in the human heart ${ }^{2}$. Ro52 $\beta$ antigen cDNA has a defective exon 4 , which encodes part of an autoepitope hotspot ${ }^{2}$.

Objectives: We investigated the clinical and laboratory characteristics of anti-Ro52 $\alpha$ and anti-Ro52 $\beta$. We also analyzed the characteristics of antiRo52 $\alpha / \beta$ within each coexisting inflammatory myositis specific autoantibody (MSA) group to reduce the influences of coexisting MSA characteristics. Methods: Among 229 dermatomyositis (DM) and polymyositis (PM) patients, 167 patients (DM 152, PM 10, juvenile DM 5) fulfilled the criteria of Bohan and Peter, and 62 clinical amyopathic DM cases fulfilled the criteria of Sontheimer. Anti-Ro52 $\alpha$ and anti-Ro52 $\beta$ antibodies were detected by ELISA.

Results: 46 of the 229 patients were anti-Ro52 $\alpha$-positive (20\%). ILD was a frequent complication in the anti-Ro52 $\alpha$-positive patients (35/42: $76 \%$ ) $(\mathrm{P}=0.0016)$, and anti-Ro52 $\alpha$ was highly positive in the anti-aminoacyl tRNA synthetase (ARS) antibody-positive group (19/32: 60\%) $(P<0.0001)$ (Table 1). However, the ILD frequencies of anti-Ro52 $\alpha$-positive and antiRo52 $\alpha$-negative patients within each group of coexisting MSA (anti-ARS, anti-TIF, anti-MDA5, etc.) were almost same $(P=1)$. The anti-Ro52 $\alpha$-positive rates were similar to the positive rates of anti-Jo1 $(61 \%)$ and other anti-ARSs (52\%) $(P=0.7)$.

Of the 46 anti-Ro52 $\alpha$-positive patients, 26 patients were anti-Ro52 $\beta$-positive. No patient was only anti-Ro52 $\beta$ positive. The anti-Ro52 $\alpha$-positive and anti-Ro52 $\beta$-positive groups were older than the anti-Ro52 $\alpha$-only-positive group $(P=0.009)$, and the average of maximum creatine kinase was higher in both of the positive groups $(\mathrm{P}=0.065)$. The 6 patients without coexisting MSA were all both anti-Ro52 $\alpha$-positive and anti-Ro52 $\beta$-positive $(P=0.03)$ (Table 2).

Conclusion: Anti-Ro52 is highly positive when anti-ARS antibodies are present, but it is not anti-Jo-1-specific. Anti-Ro52 positivity is not associated with elevated risk of ILD in any of the MSA-positive groups. The anti-Ro52 $\beta$ antibody might be an indicator of myositis.

\section{REFERENCES:}

[1] Lee AYS. A review of the role and clinical utility of anti-Ro52/TRIM21 in systemic autoimmunity. Rheumatol Int. 2017;37(8):1323-1333.

[2] Chan EK, Di Donato F, Hamel JC, Tseng CE, Buyon JP. 52-kD SS-A/Ro: genomic structure and identification of an alternatively spliced transcript encoding a novel leucine zipper-minus autoantigen expressed in fetal and adult heart. J Exp Med. 1995;182(4):983-92. 
Table 1. Clinical and laboratory characteristics with anti-Ro52 $\alpha$

\begin{tabular}{|c|c|c|c|}
\hline & $\begin{array}{c}\text { anti-Ro52 } \alpha \\
(+) \\
\mathrm{N}=46\end{array}$ & $\begin{array}{c}\text { anti-Ro52 } \alpha(- \\
) \\
N=183\end{array}$ & $P$ value \\
\hline age & $54.6 \pm 14$ & $53.1 \pm 19$ & 0.89 \\
\hline sex (female) & 35 (76\%) & $126(69 \%)$ & 0.37 \\
\hline cancer & $6(13 \%)$ & $43(23 \%)$ & 0.15 \\
\hline ILD & $32(76 \%)^{\star}$ & $78(49 \%)^{* *}$ & 0.0016 \\
\hline creatine & $974.3^{* * *}$ & $1274^{* \star \star *}$ & 0.40 \\
\hline \multicolumn{4}{|l|}{ kinase } \\
\hline \multicolumn{4}{|l|}{ MSA } \\
\hline MDA5 & 12 (26.1\%) & 36 (19.6\%) & 0.41 \\
\hline ARS & 19 (41\%) & $13(7.1 \%)$ & $<0.00001$ \\
\hline TIF $1 \gamma$ & $4(8.7 \%)$ & $37(20 \%)$ & 0.084 \\
\hline $\mathrm{Mi}-2$ & $1(2.1 \%)$ & $12(6.5 \%)$ & 0.47 \\
\hline MJ & 1 & $11(6 \%)$ & 0.46 \\
\hline SAE & 2 & $5(2.1 \%)$ & 0.63 \\
\hline $\mathrm{PM} / \mathrm{Scl}$ & 1 & $5(2.1 \%)$ & 1 \\
\hline SRP & 0 & 4 & 0.58 \\
\hline non & $6(13 \%)$ & $56(30 \%)$ & 0.016 \\
\hline
\end{tabular}

ILD: interstitial lung disease. MSA: myositis specific autoantibodies

Table 2. Clinical and laboratory characteristics in patients with anti-Ro52 $\alpha$ and anti-Ro52 $\beta$

\begin{tabular}{lccc}
\hline & $\begin{array}{c}\text { anti-Ro52 } \alpha \text { with anti-Ro52 } \beta \\
(\mathrm{N}=26)\end{array}$ & $\begin{array}{c}\text { Anti-Ro52 } \alpha \text { without anti-Ro52 } \\
(\mathrm{N}=20)\end{array}$ & P value \\
\hline age & $59.8 \pm 12.5$ & $47.85 \pm 13.5$ & 0.009 \\
sex (female) & $21(81 \%)$ & $14(70 \%)$ & 0.49 \\
cancer & $4(15 \%)$ & $2(10 \%)$ & 0.68 \\
creatine kinase & 1267.52 & 588.4 & 0.065 \\
ILD & $17(71 \%)^{*}$ & $16(89 \%)^{* *}$ & 0.25 \\
MSA & & & \\
MDA5 & 5 & 7 & 0.31 \\
ARS & 9 & 10 & 0.74 \\
TIF1 & 3 & 1 & 0.62 \\
SAE & 1 & 1 & 1 \\
Mi-2 & 1 & 0 & 1 \\
MJ & 1 & 0 & 1 \\
PM/ScL & 0 & 1 & 0.45 \\
non & 6 & 0 & 0.03 \\
\hline
\end{tabular}

*4 cases excluded with insufficient data. ${ }^{*} 2$ cases excluded.

Disclosure of Interests: None declared

DOI: 10.1136/annrheumdis-2019-eular.1898

\section{FRI0340 LONG-TERM FOLLOW-UP OF PRIMARY RAYNAUD'S PHENOMENON COHORT OF 248 PATIENTS}

Francisco Miguel Ortiz Sanjuan*, Elvira Vicens Bernabeu, Cristina

Alcañiz Escandell, Inés Cánovas Olmos, Inmaculada Chalmeta Verdejo, Marta De la Rubia Navarro, Jorge Juan Fragio-Gil, Roxana Gonzalez Mazario, Luis

Gonzalez Puig, Elena Grau García, Jose Ivorra Cortés, Isabel Martínez Cordellat, Carmen Nájera Herranz, Rosa Negueroles Albuixech, Jose Eloy Oller Rodríguez, Cristobal Pávez Perales, Jose Andrés Román Ivorra. Rheumatology Department. HUP La Fe, Valencia, Spain

Background: Raynaud's phenomenon (RP) is frequently associated with the presence of scleroderma or other connective tissue diseases (CTD). Identify the presence of secondary RP is important to perform an adequate therapeutic management and to achieve the early control of these patients. Nailfold capillaroscopy is safe, economic, easy to perform and has proven to be useful in identifying patients with secondary RP. Objectives: To assess a long-term follow-up primary RP patient's cohort. Methods: Retrospective observational study of a wide and unselected series of patients diagnosed as primary RP from a single university hospital from January 2012 to December 2018. Patients were classified as primary RP after the presence of CTD at the onset was excluded.

Results: We studied 248 patients (210 women/38 men), with a mean age of $46.3 \pm 15.2$ years (range 15-88). After a mean follow-up period of $57.2 \pm 21.6$ months, $9(3.6 \%)$ patients were diagnosed of a CTD. The remaining 239 (96.4\%) patients continued classified as primary RP. The main pathologies observed after follow-up were: Scleroderma/Systemic sclerosis $(n=3)$, Systemic Lupus Erythematosus $(n=3)$, Rheumatoid arthritis $(n=1)$, Sjogren's syndrome $(n=2)$. The main capillaroscopic patterns observed were: Normal $(n=175)$, Unspecific $(n=64)$, Scleroderma pattern $(n=3)$ and suggestive of other rheumatic diseases pattern $(n=6)$. All the patients who developed a CTD during follow-up showed changes at successive nailfold capillaroscopic examinations. 28 of 239 patients who remained primary $\mathrm{RP}$ showed minor changes at successive nailfold capillaroscopy. The main capillaroscopic changes detected on this group were: Presence of capillary tortuosity $(n=6)$, presence of a decreased capillary density (2) and the presence of capillary bleeding $(n=20)$. Antinuclear antibodies (ANA) were positive at he onset in 46 patients (16.9\%) and after follow-up in $44(17.7 \%)$ patients.

Conclusion: After a mean follow-up period next to 5 years, most of our primary RP patients remained free of CTD. A minority of our patients showed changes at nailfold capillaroscopy exam or positivity of ANA.

Disclosure of Interests: None declared

DOI: 10.1136/annrheumdis-2019-eular.5989

\section{FRI0341 LOWER URINARY TRACT SYMPTOMS PREVALENCE IN SYSTEMIC SCLEROSIS PATIENTS: RESULTS FROM A COMPREHENSIVE ANALYSIS}

Greta Pacini ${ }^{1}$, Amelia Chiara Trombetta ${ }^{1}$, Federica Goegan ${ }^{1}$, Sabrina Paolino ${ }^{1}$, Carmen Pizzorni ${ }^{1}$, Elisa Alessandri ${ }^{1}$, Massimo Patanè ${ }^{1}$, Emanuele Gotelli ${ }^{1}$ Giorgia Ferrari ${ }^{1}$, Francesco Cattelan ${ }^{1}$, Massimo Ghio ${ }^{1}$, Andrea Casabella ${ }^{1}$, Vanessa Smith2, Maurizio Cutolo'. ' IRCCS San Martino Polyclinic Hospital, Research Laboratory and Academic Unit of Clinical Rheumatology, Department of Internal Medicine, University of Genova, Genova, Italy; ${ }^{2}$ Ghent University Hospital, Department of Internal Medicine, Belgium Unit for Molecular Immunology and Inflammation, VIB Inflammation Research Center (IRC), Ghent, Belgium

Background: Lower urinary tract symptoms (LUTS) are a seldom-reported manifestation of systemic sclerosis (SSc). Although LUTS have been described in SSc patients with a higher prevalence than in the general population, no controlled studies have been reported to date [1-3].

Objectives: To compare LUTS prevalence and severity in SSc patients and in healthy subjects and mainly to explore their association with SSC clinical and diagnostic parameters.

Methods: This study evaluated 42 SSc consecutive patients (median age 61 years, range 21-85) and 50 matched healthy subjects (median age 57 years, range 28-93). SSc diagnosis was based on the 2013 American College of Rheumatology (ACR)/European League Against Rheumatism (EULAR) criteria. LUTS were assessed through validated self-reported questionnaires derived from the International Conference on Incontinence (ICIQ-FLUTS, ICIQ-MLUTS and ICIQ-OAB) [4]. General comorbidities and/ or medication potentially related to LUTS, as well as lower urinary tract infections, were evaluated in order to exclude confounding factors. Nonparametric tests and chi-squared test were performed to compare LUTS distribution, namely of urinary incontinence (UI) and overactive bladder $(\mathrm{OAB})$, in the two populations, then linear and logistic regressions were used to test the association between SSc disease and LUTS prevalence and severity. In SSc population, linear and logistic regressions were performed to test the association between LUTS and SSc variables, including nailfold videocapillaroscopy (NVC) patterns ("Early", "Active", "Late"), SSc-related autoantibodies and dual X-ray absorptiometry (DXA) parameters. Multivariate analysis was performed to adjust the associations for potential confounders. A $\mathrm{p}$ value $<0.05$ and a confidence interval $(\mathrm{Cl})$ of $95 \%$ were considered statistically significant.

Results: SSc patients showed significantly higher prevalence and severity of $\mathrm{UI}$ and $\mathrm{OAB}$ than healthy controls $(\mathrm{p}<0.005, \mathrm{p}<0.01)$. SSc was a strong predictor of LUTS, independent of demographic data, comorbidities and treatments (OR 5.57, 95\% IC 1.64-18.88). In SSc patients sarcopenia positively correlated with $\mathrm{OAB}(\mathrm{p}<0.001)$ and reduced bone mineral density (BMD) positively correlated with $\operatorname{OAB}(p<0.05)$ and $U I(p=0.001)$. UI positively correlated with anti Scl70 Abs $(p<0.05)$ and cyclosporine treatment $(p=0.001)$ and negatively with anti RNA polymerase III Abs $(p<0.05)$; OAB positively correlated with calcinosis $(p<0.005)$ and negatively with methotrexate treatment $(p<0.05)$. NVC "Active" and "Late" patterns were predominant among SSc patients presenting LUTS (both UI and $O A B$ ), although not any statistical correlation was found.

Conclusion: For the first time urinary tract involvement was found to be significantly higher in SSc patients than in healthy matched controls. In addition, sarcopenia, bone damage and calcinosis appeared significantly correlated with LUTS, suggesting a possible interplay.

\section{REFERENCES:}

[1] John G, et al. Arthritis Care Res. 2018;70:1218-27.

[2] Sanchez K, et al. Press Medicale [Internet]. 2016;45(4):e79-89.

[3] Adler BL, et al. J Rheumatol. 2018;45:1145-52.

[4] Abrams P, et al. J Urol. 2006;175:1063-6.

Disclosure of Interests: None declared 